Amy T. Waldman, MD, MSCE

Grant T. Liu, MD

Amy M. Lavery, PhD

Geraldine Liu, MA

William Gaetz, PhD

Tomas S. Aleman, MD

Brenda L. Banwell, MD

Correspondence to

Dr. Waldman:

waldman@email.chop.edu
Supplemental data at Neurology.org/nn

\section{Optical coherence tomography and visual evoked potentials in pediatric MS}

OPEN

\section{ABSTRACT}

Objective: To determine the relative ability of optical coherence tomography (OCT) and pattern-reversal visual evoked potentials ( $\mathrm{PVEPs}$ ) to detect visual pathway involvement in pediatric-onset MS.

Methods: Pediatric-onset MS participants (onset $<18$ years) and healthy controls ( $\mathrm{HCs}$ ) underwent OCT (Cirrus HD-OCT) and pVEPs. Retinal nerve fiber layer (RNFL), ganglion cell layer to inner plexiform layer (GCL-IPL), and P100 pVEP latency were measured. Generalized estimating equation models were used to compare the groups, adjusting for age and intereye correlations.

Results: Twenty-four pediatric MS participants, 14 with a history of remote ( $>6$ months) optic neuritis (ON) in one eye (8 participants) or both the eyes (6 participants), and $24 \mathrm{HCs}$ were enrolled. RNFL thinning ( $<83 \mu \mathrm{m}, 2$ SDs below $\mathrm{HC}$ eyes) occurred in $50 \%$ of ON eyes vs $5 \%$ of non-ON eyes. Prolonged VEP latency ( $>109 \mathrm{msec}$ ) occurred in $58 \%$ of ON eyes and $55 \%$ of non-ON eyes. A clinical history of ON predicted RNFL $(p<0.001)$ and GCL-IPL thinning $(p=0.011)$, whereas prolonged pVEP latency in children with MS occurred independent of ON history.

Conclusions: OCT and PVEPs provide complementary but distinct insights. OCT is sensitive to retinal changes in the context of clinical ON, whereas PVEPs are useful to detect disseminated lesions of the visual pathway in children with MS. Neurol Neuroimmunol Neuroinflamm 2017;4: e356; doi: 10.1212/NXI.0000000000000356

\section{GLOSSARY}

ETDRS = Early Treatment Diabetic Retinopathy Study; GCL-IPL = ganglion cell layer to inner plexiform layer; GEE = generalized estimating equation; HC = healthy control; OCT = optical coherence tomography; $\mathbf{O N}=$ optic neuritis; pVEP = pattern-reversal visual evoked potential; RNFL = retinal nerve fiber layer; VA = visual acuity

Optical coherence tomography (OCT) and pattern-reversal visual evoked potentials (pVEPs) assess the structural and functional integrity of the visual pathway. Although OCT and pVEPs are well-substantiated tools to evaluate MS in adults, less is known about the comparative utility of these tools in pediatric-onset MS. Importantly, because OCT interrogates retinal injury and pVEPs assess visual pathway pathology, these tools may inform differently. Prior studies evaluating VEP alone in children with optic neuritis $(\mathrm{ON})$ have demonstrated prolonged latencies and/or reduced amplitudes in $83 \%$ of children with acute ON. ${ }^{1}$ PVEPs are abnormal in $56 \%$ of children with confirmed MS at the time of their first attack, even if this attack did not include clinical ON. ${ }^{2}$ In OCT studies, thinning of the retinal nerve fiber layer (RNFL) and the ganglion cell layer (GCL) occurs after $\mathrm{ON}$, with maximal thinning in the $\mathrm{ON}$ eye recorded at 6 months post-ON. ${ }^{3}$ In adults with MS, thinning of the retina has also been documented in the absence of $\mathrm{ON}$, although thinning in non-ON eyes in pediatric MS has been variably detected. ${ }^{4-6}$ In pediatric-onset MS, the likelihood of an OCT or VEP abnormality in MS eyes,

From the Division of Neurology (A.T.W., A.M.L., G.L., B.L.B.), Neuro-ophthalmology Service (G.T.L.), Division of Ophthalmology, and Division of Radiology (W.G.), Children's Hospital of Philadelphia, PA; and Departments of Neurology (A.T.W., B.L.B., G.T.L.), Pediatrics (A.T. W., B.L.B.), and Ophthalmology (G.T.L., T.S.A.), Perelman School of Medicine, University of Pennsylvania, Philadelphia.

Funding information and disclosures are provided at the end of the article. Go to Neurology.org/nn for full disclosure forms. The Article Processing Charge was funded by the authors.

This is an open access article distributed under the terms of the Creative Commons Attribution-NonCommercial-NoDerivatives License 4.0 (CC BY-NC-ND), which permits downloading and sharing the work provided it is properly cited. The work cannot be changed in any way or used commercially without permission from the journal. 
with or without ON, has not been calculated. We sought to compare OCT and pVEPs in a cohort of pediatric-onset MS participants, with and without a history of $\mathrm{ON}$, in order to inform on the relative utility of these tests in the pediatric context.

METHODS Pediatric-onset MS participants (whose first attack occurred prior to age 18 years) were recruited for this crosssectional study from The Children's Hospital of Philadelphia (CHOP) between November, 2013, and July, 2015. Healthy controls (HCs) were recruited through local advertisement. Healthy participants without neurologic, ocular, or systemic disease were eligible provided they had a normal corrected visual acuity (VA) (defined as 47 or more letters correct using the Early Treatment Diabetic Retinopathy Study (ETDRS) chart, which is approximately 20/25 using Snellen acuity).

The diagnosis of MS was confirmed using the 2010 McDonald criteria. ${ }^{7}$ Prior ON was confirmed by history and documentation of reduced high-contrast VA, red desaturation, pain with ocular movement, and/or visual field defect. A chart review was performed to abstract the maximum VA deficit, presence of optic disc edema, color vision loss, pain with eye movements, and findings on confrontation visual field examination as recorded at the time of ON. MRI of the orbits (if available), brain, and spine were reviewed for all participants to confirm ON and MS diagnoses. The subsequent development of optic nerve pallor, as adjudicated by a neurologist or neuro-ophthalmologist, was documented. None of the participants had active acute $\mathrm{ON}$ at the time of evaluation, and all were at least 6 months from an acute attack of ON. Eyes were categorized as $\mathrm{ON}$ eyes, fellow eyes (the unaffected eye in a participant with unilateral $\mathrm{ON}$ ), or non$\mathrm{ON}$ eyes (no history of $\mathrm{ON}$ in either eye).

All study procedures occurred on the same day, beginning with the measurement of high-contrast VA using the ETDRS VA chart with the participant's own corrective lenses or glasses if needed. Although we did not perform cycloplegic refraction, all participants had their refractive error for each eye recorded using an autorefractor (VisuRef; Carl Zeiss Meditec, Inc., Dublin, CA) as a refractive error can affect OCT results. ${ }^{8,9}$ Refractive errors did not exceed 4 diopters.

Spectral domain OCT was obtained in a dark room without windows by a single trained technician using Cirrus HD-OCT (Model 5000, software version 6.5; Carl Zeiss Meditec, Inc.) at 27,000 A-scans per second. Participants underwent the Optic Disc Cube $(200 \times 200$ scans $)$ and Macular Cube $(512 \times 128)$ protocols for each undilated eye while fixating on landmarks positioned nasally or centrally to the Optic Disc Cube and Macular Cube, respectively. The technician verified that the images were focused and centered with uniform illumination and assessed for artifacts as recommended by the OSCAR-IB Consensus Criteria for Retinal OCT Quality Assessment. ${ }^{10}$ Only scans meeting these criteria and having a signal strength $\geq 7$ were used for the analysis. Segmentation was performed using the built-in automatic segmentation algorithm of the OCT system. Inspection of the resulting Macular Cube cross sections revealed no evidence for eccentric fixation as the fovea was always centered at fixation. RNFL thickness measured from an interpolated 1.7-mm-radius circle centered on the optic nerve head obtained from the Optic Disc Cube scan. GCL to inner plexiform layer thickness (GCLIPL) was obtained from analysis of the parafoveal and perifoveal retina of the Macular Cube scan.
Full-field $\left(20^{\circ}\right)$ pVEPs were performed in CHOP's Neurophysiology Laboratory by trained technicians on a Natus System (software version Nicolet EDX21.1; Natus Neurology, Pleasanton, CA) using a Queens' Square montage and following the recommendations of the International Society of Clinical Electrophysiology of Vision (ISCEV). ${ }^{11}$ Participants were positioned $1 \mathrm{~m}$ from the computer screen and asked to maintain visual fixation on a red cross in the center of an alternating checkerboard pattern of black and white squares (check size $32^{\prime}$ ) at $100 \%$ contrast. Monocular recordings were performed while the participants wore their corrective lenses (if required) and with a patch over the opposite eye. Analyses focused on the midoccipital electrode channel. The P100 latency was recorded, and the tracings were reviewed and manually corrected if needed by 2 of the investigators (A.T.W. and T.S.A.). Technician and VEP machine availability were dependent on clinical workflow and were not available for every participant.

RNFL and GCL-IPL thicknesses (in micrometers) were defined as abnormal if they were beyond $2 \mathrm{SDs}$ of the mean values obtained from HC eyes. Normality of OCT data was assessed through histograms, measures of kurtosis and skewness, and the Shapiro-Wilk W test. P100 latency was considered prolonged if the latency was $>2$ SDs above the control mean. Proportions of eyes with normal and abnormal tests were determined. The sensitivity, specificity, positive and negative predictive values of OCT and VEP measures to detect MS-ON, MS fellow, and MS non-ON eyes were calculated. Generalized estimating equation (GEE) models, adjusting for age and intereye correlations, were used to compare the groups.

Intereye differences were determined by subtracting values between the right and left eyes for RNFL, GCL-IPL, and VEP latency. Intereye differences for each participant exceeding 2 $\mathrm{SDs}$ above the mean differences for $\mathrm{HC}$ eyes were assessed and compared using a $t$ test.

Standard protocol approvals, registrations, and patient consents. The study was approved by the Institutional Review Board at The Children's Hospital of Philadelphia. Written informed consent and child assent were obtained from all participants.

RESULTS Twenty-four pediatric-onset MS participants and $24 \mathrm{HCs}$ were enrolled (table 1). Eleven additional pediatric-onset MS participants declined enrollment because of time constraints or lack of interest in research. Among the MS participants enrolled, $14(58 \%)$ had a history of ON in one eye $(\mathrm{N}=8)$ or both the eyes $(\mathrm{N}=6)$. In total, $20 \mathrm{MS}$ ON eyes, 8 MS-fellow eyes, 20 MS non-ON eyes, and $48 \mathrm{HC}$ eyes were included. Children with MS and $\mathrm{ON}$ had excellent recovery of high-contrast VA: of the 20 ON eyes, 17 (85\%) achieved a VA of 20/32 or better at the time of the last follow-up examination. Optic nerve pallor was seen in 14/20 eyes (70\%) with a history of ON. None of the fellow eye optic nerves were pale. Only one of the eyes in a child without $\mathrm{ON}$ had temporal nerve pallor.

OCT scans were excluded for failing quality control (due to movement and artifacts) including RNFL images for $1 \mathrm{HC}$ eye and GCL-IPL measurements for $1 \mathrm{HC}$ and $3 \mathrm{MS}$ eyes. Eight MS participants and 6 controls did not undergo VEP because of scheduling 
Table 1 Demographics

\begin{tabular}{|c|c|c|c|}
\hline & MS (N = 24) & $\begin{array}{l}\text { Healthy controls } \\
(\mathrm{N}=24)\end{array}$ & $p$ Value \\
\hline Mean age, y (range) & $17.5(11-24)$ & $15.4(8-22)$ & 0.046 \\
\hline Sex (F:M) & $14: 10$ & $16: 8$ & 0.105 \\
\hline Race, n (\%) & & & 0.152 \\
\hline Caucasian & 15 (62.5) & $13(56.5)$ & \\
\hline African American & 9 (37.5) & $5(21.7)$ & \\
\hline Asian & $0(0)$ & $2(8.7)$ & \\
\hline Unknown/mixed & $0(0)$ & $3(13.0)$ & \\
\hline Ethnicity, n (\%) & & & 0.259 \\
\hline Hispanic & $3(12.5)$ & $1(4.2)$ & \\
\hline Non-Hispanic & 21 (87.5) & 20 (83.3) & \\
\hline Unknown/not reported & 0 & $3(8.3)$ & \\
\hline Mean disease duration, y (range) & $2.4(0.3-7.7)$ & NA & NA \\
\hline $\begin{array}{l}\text { Mean duration from last episode } \\
\text { of optic neuritis, y (range) }\end{array}$ & $2.9(0.5-6.0)$ & NA & NA \\
\hline EDSS, median (range) & $1(0-3.5)$ & NA & NA \\
\hline
\end{tabular}

Abbreviations: EDSS $=$ Expanded Disability Status Scale; NA $=$ not applicable

constraints. Three additional VEP tracings (2 MS eyes and $1 \mathrm{HC}$ eye) were excluded because of poor fixation or movement with irregular waveforms.

RNFL values were normally distributed in MS participants and controls, whereas GCL-IPL thickness demonstrated a departure from normality based on the Shapiro-Wilk W test (table e-1 and figure e-1 at Neurology.org/nn). Mean and SD values for RNFL and GCL-IPL thicknesses and VEP latencies for MS participants and HCs are listed in table 2. In the 48 MS eyes, $27 \%$ met our criteria for RNFL abnormality (thickness $<83 \mu \mathrm{m}$ ). The proportions of participants with abnormal metrics stratified by whether the eye was affected by $\mathrm{ON}$ are presented in table 3. Among those participants who completed OCT and VEP, only 4 of 30 (13\%) MS eyes were deemed abnormal by all 3 measures (RNFL thinning,
GCL-IPL thinning, and delayed VEP latencies), and 3 of these 4 eyes had a remote history of ON.

In MS participants, the presence of ON pallor $(\mathrm{N}=15$ eyes) was associated with a greater likelihood of RNFL thinning (mean $80 \mu \mathrm{m}$, SD 13) compared with RNFL thickness in nonpale eyes $(\mathrm{N}=33$ eyes; mean RNFL $94 \mu \mathrm{m}$, SD 13; $p<0.001)$. GCL-IPL was also thinner in pale optic nerves (mean $70 \mu \mathrm{m}$, $S D$ 10) compared with nonpale optic nerves (mean 79 , SD $8 ; p=0.002$ ). VEP latency, however, did not differ as a function of optic nerve pallor.

Table 4 compares the sensitivity, specificity, positive predictive value, and negative predictive value for OCT and pVEPs in MS-ON and non-ON eyes compared with $\mathrm{HC}$ eyes. RNFL thinning has moderate sensitivity $(50 \%)$ for the detection of MS-ON eyes but not for the detection of MS in the absence of ON (5\%). GCL-IPL thinning was not sensitive for detecting MS in any of the groups. Although the specificity and positive predictive values of GCL-IPL thickness were high $(100 \%)$, this was driven by the absence of GCL-IPL thinning in the HCs. The sensitivity, specificity, and positive and negative predictive values for VEPs were similar in MS eyes regardless of ON history, indicating that pVEPs are supportive of an MS diagnosis but not helpful in determining prior $\mathrm{ON}$.

By defining a cut-off for abnormal retinal thickness and VEP latencies, which was based on 2 SDs below the normative values, it is possible to miss significant loss or slowing in an individual whose results are asymmetric but fall within 1-2 SD of the mean. We explored intereye differences for OCT and VEP results to determine whether our definition of abnormal (2 SDs below the mean) failed to detect potentially more subtle differences in pediatric-onset MS eyes. Intereye differences for RNFL and GCL-IPL thicknesses and VEP latency for HC and MS participants are presented in table 5. We defined abnormal intereye differences as the mean intereye difference plus 2 SDs from HC (RNFL $6 \mu \mathrm{m}$, GCL-IPL

Table 2 Mean OCT and VEP values

\begin{tabular}{|c|c|c|c|c|c|c|c|c|c|}
\hline & \multirow[b]{2}{*}{$\begin{array}{l}\text { Healthy } \\
\text { eyes }(N=48)\end{array}$} & \multicolumn{8}{|l|}{ MS eyes } \\
\hline & & All eyes $(N=48)$ & $\begin{array}{l}\text { p Value } \\
\text { vs control } \\
\text { eyes }\end{array}$ & $\begin{array}{l}\text { Non-ON eyes } \\
(\mathrm{N}=20)\end{array}$ & $\begin{array}{l}\text { p Value } \\
\text { vs control } \\
\text { eyes }\end{array}$ & $\begin{array}{l}\text { Fellow eyes } \\
(\mathrm{N}=8)\end{array}$ & $\begin{array}{l}\text { p Value } \\
\text { vs control } \\
\text { eyes }\end{array}$ & ON eyes $(\mathrm{N}=20)$ & $\begin{array}{l}\text { p Value } \\
\text { vs contro } \\
\text { eyes }\end{array}$ \\
\hline RNFL (SD) & $100(8), N=47$ & $90(14), N=48$ & 0.002 & $100(10), N=20$ & 0.193 & $87(12), N=8$ & $<0.001$ & $81(12), N=20$ & $<0.001$ \\
\hline GCL-IPL (SD) & $83(6), N=45$ & $76(9), N=46$ & 0.001 & $79(9), N=20$ & 0.103 & 77 (10), $N=7$ & 0.032 & $72(9), N=18$ & $<0.001$ \\
\hline VEP latency (SD) ${ }^{b}$ & $101(4), N=35$ & 115 (17), $N=30$ & $<0.001$ & $119(17), N=11$ & $<0.001$ & $108(6), N=7$ & $<0.001$ & $115(20), N=12$ & 0.001 \\
\hline
\end{tabular}

Abbreviations: GCL-IPL = ganglion cell layer to inner plexiform layer; OCT = optical coherence tomography; ON = optic neuritis; $\mathrm{pVEP}=$ pattern-reversal visual evoked potential; RNFL = retinal nerve fiber layer.

${ }^{a}$ Generalized estimating equation models were used to compare each group with healthy eyes, adjusting for age and intereye correlations.

${ }^{b}$ The clinical features of the MS participants who underwent pVEPs $(N=30$ eyes) did not differ from the patients who did not complete this test $(N=18$ eyes). There was no difference between the groups with respect to the number of ON eyes $(p=0.580)$, the age at testing $(p=0.811)$, disease duration $(p=$ 0.937), or mean high-contrast letter acuity scores $(p=0.1435)$. 
Table 3 Proportions with abnormal findings

$\begin{array}{llllll}\text { Proportions of patients with } & \text { HC eyes }(\mathbf{N}=48) & \text { All eyes } & \text { Non-ON eyes } & \text { Fellow eyes } & \text { ON eyes } \\ \text { Visual acuity deficit } & 0 & 7 / 48=15 \% & 3 / 20=15 \% & 1 / 8=13 \% & 3 / 20=15 \% \\ \text { Pallor present } & 0 & 15 / 48=31 \% & 1 / 20=5 \% & 0 & 14 / 20=70 \% \\ \text { RNFL abnormal }(<83 \mu \mathrm{m}) & 2 / 47=4 \% & 13 / 48=27 \% & 1 / 20=5 \% & 1 / 7=13 \% & 10 / 20=50 \% \\ \text { GCL-IPL abnormal }(<71 \mu \mathrm{m}) & 0 & 10 / 45=22 \% & 2 / 20=10 \% & 2 / 8=25 \% & 6 / 18=33 \% \\ \begin{array}{l}\text { VEP latency prolonged } \\ (>109 \text { msec) }\end{array} & 2 / 35=6 \% & 16 / 30=53 \% & 6 / 11=55 \% & 3 / 7=43 \% & 7 / 12=58 \%\end{array}$

Abbreviations: GCL-IPL = ganglion cell layer to inner plexiform layer; $\mathrm{HC}=$ healthy control; $\mathrm{ON}=$ optic neuritis; $\mathrm{RNFL}=$ retinal nerve fiber layer; VEP = visual evoked potential.

One HC had normal high-contrast visual acuity (47 letters correct in each eye) but had an RNFL of 82 and $81 \mu \mathrm{m}$ in the right and left eyes. The GCL-IPL and VEP latencies for this individual were within the normal range. One HC had prolonged VEP latencies (112 and $116 \mathrm{msec}$ in the right and left eyes), despite normal high-contrast visual acuity (59 and 60 letters), RNFL, and GCL-IPL thicknesses.

$2 \mu \mathrm{m}$, and pVEP latency $4 \mathrm{msec}$ ). Intereye differences greater than $6 \mu \mathrm{m}$ for RNFL thickness occurred in 10 MS participants, 8 of whom had a history of $\mathrm{ON}$ ( 5 with unilateral $\mathrm{ON}$ and 3 with bilateral $\mathrm{ON}$ ); an intereye difference greater than $2 \mu \mathrm{m}$ in GCL-IPL occurred in $11 \mathrm{MS}$ participants, 8 of whom had ON ( 5 with unilateral $\mathrm{ON}$ and 3 with bilateral $\mathrm{ON}$ ), and intereye difference in VEP latency greater than $4 \mathrm{msec}$ occurred in $9 \mathrm{MS}$ participants, 6 of whom had ON (4 with unilateral, 2 with bilateral $\mathrm{ON})$. Of note, if intereye difference criteria were used to define optic nerve involvement by OCT, $3 \mathrm{MS}$ patients with clinical histories of $\mathrm{ON}$ would have been identified whose RNFL or GCL-IPL measures failed to meet the absolute cut-off criteria for abnormality $(<2$ SDs below the control mean) (see figure e-2 for a representative participant). Furthermore, in $3 \mathrm{MS}$ additional patients, intereye OCT measures suggested relative (but not absolute) thinning in one eye in the absence of any history of $\mathrm{ON}$.

A clinical history of $\mathrm{ON}$ predicted RNFL thinning $(p<0.001)$ using regression analysis. After accounting for $\mathrm{ON}$ and intereye correlations in GEE models, OCT parameters did not differ between MS eyes and
HCs for RNFL thickness $(p=0.565)$ or GCL-IPL thickness $(p=0.051)$. Prolonged VEP latency occurred independent of ON history $(p<0.001)$. For MS eyes, the presence of pallor in an eye predicted RNFL thinning $(p<0.001)$ and GCL-IPL thinning $(p=0.001)$ but not VEP latency $(p=$ $0.159)$. When $\mathrm{ON}$ and pallor were both included in the model which adjusted for age and intereye correlations, there was no difference in RNFL thickness between MS patient and HC eyes $(p=0.607)$ or GCL-IPL thickness (0.061). VEP latency differed between MS and control eyes, even after accounting for ON and pallor $(p=0.001)$.

DISCUSSION OCT and VEP provide valuable, but different information in pediatric-onset patients with MS. OCT confirms the history of ON, but unlike the findings in adult MS, OCT infrequently identifies retinal abnormalities in pediatric-onset patients with MS who have not experienced clinical evidence of optic nerve involvement. Only $5 \%$ of our patients with MS demonstrated RNFL thinning and $10 \%$ had GCL-IPL thinning in the absence of prior ON. By contrast, prolonged pVEP latency occurred

Table 4 Capacity of OCT and VEP to correctly identify MS eyes with and without ON compared with healthy eyes

\begin{tabular}{|c|c|c|c|c|c|c|c|c|c|}
\hline & \multicolumn{3}{|c|}{ MS-ON eyes compared with $\mathrm{HC}$ eyes } & \multicolumn{3}{|c|}{ MS fellow eyes (non-ON eye) vs HCs } & \multicolumn{3}{|c|}{ MS non-ON eyes vs healthy controls } \\
\hline Specificity & 96 & 100 & 94 & 96 & 100 & 94 & 96 & 100 & 94 \\
\hline $\begin{array}{l}\text { Positive predictive } \\
\text { value }\end{array}$ & 83 & 100 & 78 & 50 & 100 & 60 & 33 & 100 & 75 \\
\hline
\end{tabular}

Abbreviations: GCL-IPL = ganglion cell layer to inner plexiform layer; $\mathrm{HC}=$ healthy control; OCT = optical coherence tomography; $\mathrm{ON}=$ optic neuritis; $\mathrm{RNFL}=$ retinal nerve fiber layer; VEP = visual evoked potential. 
Table 5 Intereye differences for OCT and VEP metrics

\begin{tabular}{|llll} 
& HC & MS & $p$ Value \\
\hline RNFL, mean difference, $\mu \mathrm{m}$ & 2.3 (SD 1.7) [range 0-6] & 7.6 (SD 7.1) [range 0-30] & 0.0011 \\
\hline GCL-IPL, mean difference, $\mu \mathrm{m}$ & 0.7 (SD 0.8) [range 0-2] & 5.1 (SD 6.0) [range 0-24] & 0.0012 \\
VEP latency, mean difference, msec & 1.5 (SD 1.3) [range 0.2-5] & 13.3 (SD 13.6) [range 0.8-45.4] & 0.0012
\end{tabular}

Abbreviations: GCL-IPL = ganglion cell layer to inner plexiform layer; $\mathrm{HC}=$ healthy control; $\mathrm{OCT}=$ optical coherence tomography; RNFL = retinal nerve fiber layer; VEP = visual evoked potential.

in $53 \%$ of patients with MS, a finding that was not influenced by a history of ON.

Our data are aligned with adult studies in which VEP abnormalities are more frequently seen than RNFL thinning in non-ON eyes. ${ }^{12,13}$ Our VEP results are also similar to a prior study in which in 48 of the 85 (56\%) pediatric patients with MS demonstrated VEP prolongation more than 2.5 SDs beyond control mean latencies and/or reduced amplitudes. ${ }^{2}$ Also in line with our findings, another study of 14 pediatric patients with MS reported no difference in VEP latencies between MS-ON eyes and MS nonON eyes $(p=0.524)$ or between MS-ON eyes and MS fellow eyes $(p=0.654) .^{5}$

OCT has been reported in 54 pediatric patients with MS collectively between 3 prior studies, all of which reported a significant decrease in mean RNFL thickness among MS-ON eyes compared with HC eyes. ${ }^{4-6}$ In this study, we demonstrated a mean reduction of $19 \mu \mathrm{m}$ in RNFL thickness among pediatric MS-ON eyes vs $\mathrm{HCs}$, which is comparable with a meta-analysis of adult OCT data which revealed a mean RNFL loss of $20.38 \mu \mathrm{m}$ (95\% CI -22.86 to -17.91$)$ among $956 \mathrm{MS}$ ON eyes vs $1,107 \mathrm{HC}$ eyes. ${ }^{14}$

Although the comparison of mean scores enables the identification of population differences, such data do not necessarily inform on the utility of testing for the individual patient. In our cohort, only $50 \%$ of children with MS and a history of $\mathrm{ON}$ will have reduced RNFL that is 2 SDs below the mean for HCs; this proportion of children with abnormal RNFL results in MS-ON eyes is substantiated by 2 previous pediatric MS OCT studies based on their published mean data. ${ }^{4,6}$ Although OCT parameters in half of the MS-ON eyes may fall within a normal reference range, intereye differences in RNFL and GCL-IPL thicknesses may help the clinician in identifying a remote history of $\mathrm{ON}$.

In this study, the mean RNFL thickness in MS non-ON eyes was equal to the mean of HCs. We did not replicate findings of 2 other OCT studies of pediatric MS participants in which mean RNFL thickness was reduced in non-ON eyes, findings that were interpreted as supportive of a more degenerative global MS pathology. ${ }^{4,5}$ Using intereye differences in
OCT measures, we did identify 3 patients with MS without a history of ON whose intereye differences exceeded the intereye variability seen in our control cohort. However, 2 of these 3 patients had RNFL and GCL-IPL values above the normative mean (despite the intereye differences), rendering it difficult to propose evidence of non-ON global MS-related injury in our cohort.

There are several important caveats to our work. The rarity of pediatric MS limits sample size. We did not perform formal cycloplegic refractions, an important issue given that severe myopia is associated with a thinner retina, and VA testing and VEPs ideally should be performed with the patient's best refractive correction. To mitigate this concern at least in part, we used an autorefractor to estimate the refractive error and none of the patients had severe myopia $(>-4.00)$. Furthermore, nearly all of our patients with MS had visual acuities near or equal to 20/40, and all HCs by inclusion criteria had 20/ $20 \mathrm{VA}$ in each eye, making even mild uncorrected myopia unlikely to have been a factor in this study.

These results can inform clinical decision making. For a patient with suspected or confirmed MS who describes a remote history of vision loss, an abnormal OCT (defined either by absolute or intereye measures that deviate substantially from normative values) may be used to support a prior episode of ON. However, a normal OCT does not exclude the possibility of prior ON. VEP abnormalities detect dissemination of disease in patients being investigated for MS and are informative even in the absence of $\mathrm{ON}$.

\section{AUTHOR CONTRIBUTIONS}

Amy T. Waldman: study concept/design, acquisition of data, statistical analysis, interpretation of data, drafting/revising the manuscript for content, including medical writing for content and obtaining funding, and study supervision and coordination. Grant T. Liu: interpretation of data, drafting/revising the manuscript for content, including medical writing for content. Amy M. Lavery: acquisition of data, analysis and interpretation of data, statistical analysis, and drafting/revising the manuscript for content, including medical writing for content. Geraldine Liu: acquisition of data and drafting/revising the manuscript for content, including medical writing for content. William Gaetz, Tomas S. Aleman, and Brenda L. Banwell: interpretation of data and drafting/revising the manuscript for content, including medical writing for content.

\section{STUDY FUNDING}

This study was supported by the NIH (NINDS) (K23NS069806, PI: A. Waldman). 


\section{DISCLOSURE}

A.T. Waldman received travel funding from Novartis; receives publishing royalties from UpToDate; has consulted for Optum; received research support from Biogen and NIH/National Institute of Neurological Disorders and Stroke; and holds stock or stock options in Pfizer and Spark Therapeutics. G.T. Liu serves on the editorial board for Journal of Neuro-Ophthalmology; receives royalties from Elsevier. A.M. Lavery reports no disclosures. G. Liu's spouse receives royalties from Elsevier. W. Gaetz reports no disclosures. T.S. Aleman received research support from NIH. B.L. Banwell served on the scientific advisory board for Biogen, Sanofi, Eli Lilly, and Novartis; received travel funding and/or speaker honoraria from Biogen, Merck Serono, Teva, and Bayer; serves on the editorial board for Neurology ${ }^{\circledR}$; consulted for Biogen Idec, Eli Lilly, Sanofi, and Novartis; has spoken at an event supported by the Consortium of MS Centers; and received research support from Multiple Sclerosis Society of Canada, Multiple Sclerosis Scientific Research Foundation, and National Multiple Sclerosis Society. Go to Neurology.org/nn for full disclosure forms.

Received January 27, 2017. Accepted in final form March 24, 2017.

\section{REFERENCES}

1. Tekavcic-Pompe M, Stirn-Kranjc B, Brecelj J. Optic neuritis in children-clinical and electrophysiological followup. Doc Ophthalmol 2003;107:261-270.

2. Pohl D, Rostasy K, Treiber-Held S, et al. Pediatric multiple sclerosis: detection of clinically silent lesions by multimodal evoked potentials. J Pediatr 2006;149: $125-127$

3. Costello F, Hodge W, Pan YI, Eggenberger E, Coupland S, Kardon RH. Tracking retinal nerve fiber layer loss after optic neuritis: a prospective study using optical coherence tomography. Mult Scler 2008;14:893-905.

4. Yeh EA, Weinstock-Guttman B, Lincoff N, et al. Retinal nerve fiber thickness in inflammatory demyelinating diseases of childhood onset. Mult Scler 2009;15:802-810.
5. Yilmaz U, Gucuyener K, Erin DM, et al. Reduced retinal nerve fiber layer thickness and macular volume in pediatric multiple sclerosis. J Child Neurol 2012;27:1517-1523.

6. Waldman AT, Hiremath G, Avery RA, et al. Monocular and binocular low-contrast visual acuity and optical coherence tomography in pediatric multiple sclerosis. Mult Scler Relat Disord 2014;3:326-334.

7. Polman $\mathrm{CH}$, Reingold SC, Banwell B, et al. Diagnostic criteria for multiple sclerosis: 2010 revisions to the McDonald criteria. Ann Neurol 2011;69:292-302.

8. Samarawickrama C, Wang XY, Huynh SC, Burlutsky G, Stapleton F, Mitchell P. Effects of refraction and axial length on childhood optic disk parameters measured by optical coherence tomography. Am J Ophthalmol 2007; 144:459-461.

9. El-Dairi MA, Asrani SG, Enyedi LB, Freedman SF. Optical coherence tomography in the eyes of normal children. Arch Ophthalmol 2009;127:50-58.

10. Tewarie P, Balk L, Costello F, et al. The OSCAR-IB consensus criteria for retinal OCT quality assessment. PLoS One 2012;7:1-7.

11. Odom JV, Bach $M$, Brigell $M$, et al. ISCEV standard for clinical visual evoked potentials: (2016 update). Doc Ophthalmol 2016;133:1-9.

12. Naismith RT, Tutlam NT, Xu J, et al. Optical coherence tomography is less sensitive than visual evoked potentials in optic neuritis. Neurology 2009;73:46-52.

13. Di Maggio G, Santangelo R, Guerrieri S, et al. Optical coherence tomography and visual evoked potentials: which is more sensitive in multiple sclerosis? Mult Scler 2014;20: 1342-1347.

14. Petzold A, de Boer JF, Schippling S, et al. Optical coherence tomography in multiple sclerosis: a systematic review and meta-analysis. Lancet Neurol 2010;9:921-932. 


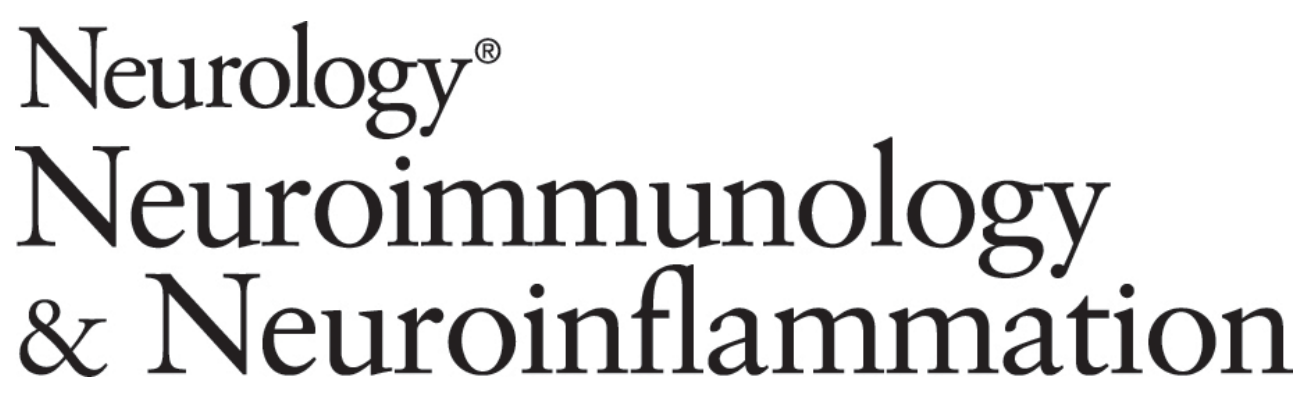

Optical coherence tomography and visual evoked potentials in pediatric MS

Amy T. Waldman, Grant T. Liu, Amy M. Lavery, et al.

Neurol Neuroimmunol Neuroinflamm 2017;4;

DOI 10.1212/NXI.0000000000000356

This information is current as of June 5, 2017

Neurol Neuroimmunol Neuroinflamm is an official journal of the American Academy of Neurology.

Published since April 2014, it is an open-access, online-only, continuous publication journal. Copyright

Copyright $\odot 2017$ The Author(s). Published by Wolters Kluwer Health, Inc. on behalf of the American

Academy of Neurology.. All rights reserved. Online ISSN: 2332-7812.

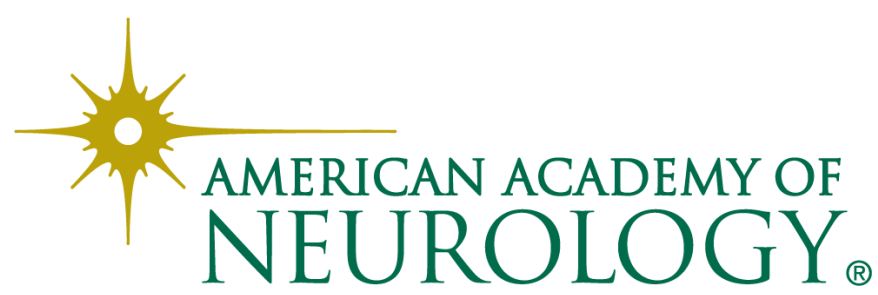




\section{Updated Information \&} Services

\section{Supplementary Material}

References

Subspecialty Collections

Permissions \& Licensing

Reprints including high resolution figures, can be found at: http://nn.neurology.org/content/4/4/e356.full.html

Supplementary material can be found at: http://nn.neurology.org/content/suppl/2017/06/26/4.4.e356.DC1

This article cites 14 articles, 0 of which you can access for free at: http://nn.neurology.org/content/4/4/e356.full.html\#\#ref-list-1

This article, along with others on similar topics, appears in the following collection(s):

\section{All Pediatric}

http://nn.neurology.org//cgi/collection/all_pediatric

Evoked Potentials/Visual

http://nn.neurology.org//cgi/collection/evoked_potentials-visual

Multiple sclerosis

http://nn.neurology.org//cgi/collection/multiple_sclerosis

Optic neuritis; see Neuro-ophthalmology/Optic Nerve

http://nn.neurology.org//cgi/collection/optic_neuritis

Information about reproducing this article in parts (figures,tables) or in its entirety can be found online at:

http://nn.neurology.org/misc/about.xhtml\#permissions

Information about ordering reprints can be found online:

http://nn.neurology.org/misc/addir.xhtml\#reprintsus

Neurol Neuroimmunol Neuroinflamm is an official journal of the American Academy of Neurology.

Published since April 2014, it is an open-access, online-only, continuous publication journal. Copyright

Copyright $\odot 2017$ The Author(s). Published by Wolters Kluwer Health, Inc. on behalf of the American

Academy of Neurology.. All rights reserved. Online ISSN: 2332-7812.

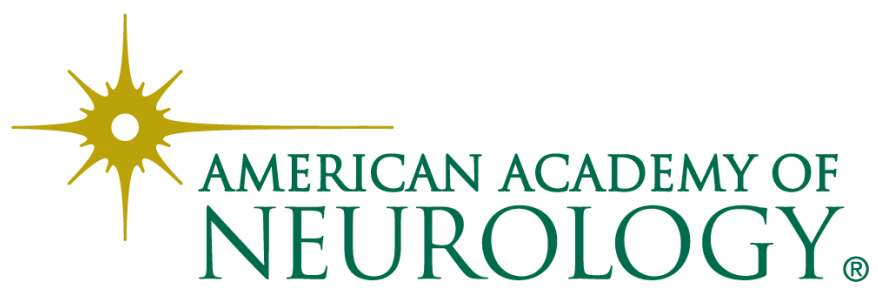

